\title{
EXTREMELY RED COMPACT RADIO SOURCES: THE EMPTY FIELD OBJECTS
}

\author{
C. A. Beichman, ${ }^{1,2}$ S. H. Pravdo, ${ }^{1,2}$ G. Neugebauer,${ }^{3}$ B. T. Soifer, ${ }^{3}$ K. Matthews, ${ }^{3}$ \\ AND H. A. WOOTTEN, ${ }^{1,4}$ \\ Received 1980 November 24; accepted 1981 February 9
}

\begin{abstract}
$10 \mu \mathrm{m}$ radiation has been detected from $1413+135$, one of the very red objects discovered by Rieke, Lebofsky, and Kinman at near-infrared wavelengths. The spectrum of this object flattens at wavelengths longer than $2.2 \mu \mathrm{m}$. Upper limits are also given for the $10 \mu \mathrm{m}$ emission from $2255+14$, $0026+34$, and $0406+121$. Photometry between 1.25 and $2.2 \mu$ m confirms the variability of $1413+135$, $2255+41$, and $0406+121$. Five percent resolution spectra of $1413+135$ and $0406+121$ between 1.5 and $2.4 \mu \mathrm{m}$ show no emission or absorption lines. The spectral data rule out the possibility that $1413+135$ is a quasar with normal line strengths and a redshift $1.3>z>4$. The lack of features of the 1.5-2.4 $\mu \mathrm{m}$ spectra, the rapid variability, and the overall shape of the radio, infrared, and X-ray energy distributions are consistent with a BL Lac nature for these objects.
\end{abstract}

Subject headings: BL Lacertae objects - infrared: sources - quasars

\section{INTRODUCTION}

Rieke, Lebofsky, and Kinman (1979, hereafter RLK) discovered relatively strong near-infrared emission from flat-spectrum radio sources located in fields in which no visual counterparts had been identified. Subsequent observations have shown that these radio sources do indeed have optical counterparts, but ones with a visual magnitude fainter than $\sim 19.5$. In about half of the "empty fields" they observed, RLK found infrared sources with very red energy distributions; between 0.5 and $2.2 \mu \mathrm{m}$ the spectral indices, $\alpha$, (defined by $\left.S_{v} \propto v^{\alpha}\right)$, are about -3 . Some of the sources showed significant variablity on time scales as short as 3 weeks. The extreme redness of the energy distributions led RLK to suggest that these sources might be members of a new class of quasars.

Since one of the most striking features of these objects is the steepness of their energy distributions from the visual to the near-infrared, it is important to determine whether or not such a steep rise continues out to longer wavelengths. In this paper we show that the energy distribution of at least one of these objects flattens at wavelengths longer than $2.2 \mu \mathrm{m}$, approaching a distribution characteristic of BL Lac objects.

\section{OBSERVATIONS}

Infrared observations were made using both the $3 \mathrm{~m}$ NASA Infrared Telescope Facility (IRTF) at Mauna Kea, Hawaii, and the $5 \mathrm{~m}$ Hale telescope at Mount

\footnotetext{
${ }^{1}$ California Institute of Technology, Division of Physics, Mathematics and Astronomy.

${ }^{2}$ Visiting Astronomer, Infrared Telescope Facility, operated by the University of Hawaii under contract with the National Aeronautics and Space Administration.

${ }^{3}$ Palomar Observatory, California Institute of Technology.

4 Owens Valley Radio Observatory, California Institute of Technology.
}

Palomar. A journal of the observations is given in Table 1. The IRTF Ge:Ga bolometer was used with a 6 " focal plane aperture and a $5 \mu \mathrm{m}$ wide filter centered at $10.6 \mu \mathrm{m}$. The measurements with the $5 \mathrm{~m}$ telescope were made using a bolometer with a 4 " focal plane aperture and a filter similar to that used at the IRTF. Observations with $J(1.25 \mu \mathrm{m}), H(1.65 \mu \mathrm{m})$, and $K(2.2 \mu \mathrm{m})$ filters were made using InSb detectors and focal plane apertures of $6^{\prime \prime}$ and $5 "$ at IRTF and Palomar. A 5\% resolution filter wheel was used at the $5 \mathrm{~m}$ telescope on 1980 February 11 (UT) to obtain spectra of $1413+135$ and $0406+121$ between 1.5 and $2.4 \mu \mathrm{m}$.

The sources were located by offsets from nearby stars. If RLK listed both radio and optical positions, the latter were used. Offsets between neighboring stars showed that offsets at both the IRTF and the $5 \mathrm{~m}$ telescope were more accurate than 0.5 over $30^{\prime}$. At the $5 \mathrm{~m}$ telescope it was possible to see and set the telescope directly on all of the sources using the TV acquisition camera. Within the uncertainties of setting the telescope, no significant discrepancies were found between the optical and radio positions.

In the case of $2255+41$, there is a field star, fainter than $0.2 \mathrm{mJy}$ at $2.2 \mu \mathrm{m}$, located about 2.5 south of the object, i.e., just at the edge of the focal plane aperture. Although care was taken to exclude this star from the beam, the effects of variable seeing and/or poor guiding could have resulted in the contamination of the measurement of $2255+41$ at $1.25 \mu \mathrm{m}$ and $1.65 \mu \mathrm{m}$, if the star is of an early spectral type.

The infrared observations were calibrated with respect to the Caltech standards (Elias and Neugebauer 1981); the conversion from magnitudes to flux densities is listed in Neugebauer et al. (1979). A correction has been made to the $10 \mu \mathrm{m}$ fluxes for the effect of the different energy distributions of sources relative to those of the calibration stars. A spectral index of -1.5 was assumed for the 
TABLE 1

LOG OF INFRARED OBSERVATIONS

\begin{tabular}{|c|c|c|c|c|c|c|c|c|}
\hline \multirow[b]{2}{*}{ Object } & \multirow[b]{2}{*}{ Obs. $^{c}$} & \multirow[b]{2}{*}{ Date (UT) } & \multicolumn{4}{|c|}{ Flux Density $(\mathrm{mJy})^{\mathrm{a}}$} & \multicolumn{2}{|c|}{ Spectral Indices ${ }^{\mathrm{b}}$} \\
\hline & & & $1.25 \mu \mathrm{m}$ & $1.65 \mu \mathrm{m}$ & $2.2 \mu \mathrm{m}$ & $10.6 \mu \mathrm{m}$ & $\alpha_{H K}$ & $\alpha_{J H}$ \\
\hline \multirow[t]{9}{*}{$1413+135 \ldots \ldots \ldots \ldots \ldots$} & UA & 1979 Feb 10 & $0.46 \pm 0.10$ & $1.17 \pm 0.10$ & $3.32 \pm 0.10$ & $\cdots$ & $-3.6 \pm 0.3$ & $-3.4 \pm 0.8$ \\
\hline & UA & 1979 Mar 07 & $\ldots$ & $\ldots$ & $1.72 \pm 0.12$ & $\ldots$ & $\ldots$ & $\ldots$ \\
\hline & UA & 1979 Mar 08 & $\cdots$ & $\cdots$ & $1.98 \pm 0.18$ & $\ldots$ & $\cdots$ & $\cdots$ \\
\hline & $\mathbf{P}$ & 1980 Feb 10 & $0.24 \pm 0.07$ & $1.01 \pm 0.07$ & $2.55 \pm 0.20$ & $\ldots$ & $-3.3 \pm 0.4$ & $-5.2 \pm 1.1$ \\
\hline & $\mathbf{P}$ & 1980 Jul 21 & $0.30 \pm 0.06$ & $0.71 \pm 0.04$ & $1.71 \pm 0.09$ & $\ldots$ & $-3.1 \pm 0.3$ & $-3.1 \pm 0.8$ \\
\hline & MK & 1980 Jul 24 & $\ldots$ & $\ldots$ & & $31 \pm 10$ & ... & $\ldots$ \\
\hline & MK & $1980 \mathrm{Jul} 25$ & $\ldots$ & $\ldots$ & $2.15 \pm 0.20$ & $27 \pm 11$ & & \\
\hline & $\mathbf{P}$ & 1980 Aug 04 & $0.15 \pm 0.06$ & $0.71 \pm 0.03$ & $1.98 \pm 0.10$ & $\ldots$ & $-3.6 \pm 0.2$ & $-5.6 \pm 1.5$ \\
\hline & $\mathbf{P}$ & 1980 Aug 05 & $0.24 \pm 0.10$ & $0.66 \pm 0.03$ & $1.76 \pm 0.14$ & $\ldots$ & $-3.4 \pm 0.3$ & $-3.6 \pm 1.5$ \\
\hline \multirow[t]{5}{*}{$2255+41 \ldots \ldots \ldots \ldots \ldots \ldots$} & UA & 1978 Nov 19 & $\ldots$ & $0.18 \pm 0.06$ & $0.33 \pm 0.07$ & .. & $-2.1 \pm 1.4$ & $\ldots$ \\
\hline & $\mathbf{P}$ & 1980 Jul 21 & $<0.3$ & $0.13 \pm 0.03$ & $0.41 \pm 0.07$ & $\cdots$ & $-4.0 \pm 1.0$ & $\cdots$ \\
\hline & MK & 1980 Jul 24 & $\ldots$ & $\ldots$ & $\ldots$ & $<23$ & $\cdots$ & $\cdots$ \\
\hline & $\mathbf{P}$ & 1980 Aug 04 & $0.21 \pm 0.03$ & $0.29 \pm 0.02$ & $0.40 \pm 0.05$ & $\ldots$ & $-1.1 \pm 0.5$ & $-1.2 \pm 0.6$ \\
\hline & $\mathbf{P}$ & 1980 Oct 05 & $0.23 \pm 0.04$ & $0.30 \pm 0.02$ & $0.34 \pm 0.04$ & $\ldots$ & $-0.4 \pm 0.5$ & $-1.0 \pm 0.7$ \\
\hline \multirow{3}{*}{$0026+34 \ldots \ldots \ldots \ldots \ldots \ldots$} & MK & 1980 Jul 24 & $\ldots$ & & & $<27$ & & $\cdots$ \\
\hline & $\mathbf{P}$ & 1980 Aug 04 & $0.26 \pm 0.09$ & $0.25 \pm 0.02$ & $0.39 \pm 0.03$ & $\ldots$ & $-1.5 \pm 0.4$ & $+0.1 \pm 1.3$ \\
\hline & $\mathbf{P}$ & 1980 Oct 05 & $0.16 \pm 0.04$ & $0.29 \pm 0.02$ & $0.39 \pm 0.04$ & $\ldots$ & $-1.0 \pm 0.4$ & $-2.1 \pm 0.9$ \\
\hline \multirow[t]{8}{*}{$0406+121 \ldots \ldots \ldots \ldots \ldots$} & UA & 1978 Aug 28 & $0.45 \pm 0.11$ & $0.78 \pm 0.09$ & $1.32 \pm 0.16$ & ... & $-1.8 \pm 0.6$ & $-2.0 \pm 1.0$ \\
\hline & UA & 1978 Sep 11 & $\ldots$ & $0.39 \pm 0.13$ & $1.18 \pm 0.20$ & & $-3.8 \pm 1.3$ & $\ldots$ \\
\hline & UA & 1979 Feb 10 & $0.17 \pm 0.07$ & $0.44 \pm 0.08$ & $0.69 \pm 0.08$ & $\ldots$ & $-1.6 \pm 0.8$ & $-3.4 \pm 1.6$ \\
\hline & UA & 1979 Mar 08 & $\ldots$ & $\ldots$ & $<0.35$ & $\ldots$ & $\ldots$ & $\ldots$ \\
\hline & $\mathbf{P}$ & 1980 Feb 10 & $\ldots$ & $0.32 \pm 0.03$ & $0.39 \pm 0.04$ & $\ldots$ & $-0.7 \pm 0.7$ & \\
\hline & $\mathbf{P}$ & 1980 Oct 04 & $0.15 \pm 0.04$ & $0.38 \pm 0.03$ & $0.55 \pm 0.05$ & $<32$ & $-1.3 \pm 0.4$ & $-3.4 \pm 1.0$ \\
\hline & $\mathbf{P}$ & 1980 Oct 05 & $0.20 \pm 0.04$ & $0.36 \pm 0.03$ & $0.57 \pm 0.06$ & $\ldots$ & $-1.6 \pm 0.5$ & $-2.1 \pm 0.8$ \\
\hline & $\mathbf{P}$ & 1980 Oct 06 & $\ldots$ & $0.33 \pm 0.03$ & $0.57 \pm 0.06$ & $\ldots$ & $-1.9 \pm 0.5$ & $\ldots$ \\
\hline
\end{tabular}

all limits are $3 \sigma$.

b Spectral index $\alpha$ defined by $S_{v} \propto v^{\alpha}$.

c $\mathrm{UA}=$ University of Arizona Observatory data from RLK, P = Palomar, MK = Mauna Kea.

sources, although the effect of assuming different indices is small relative to the statistical uncertainties. The sources are sufficiently faint that the dominant source of uncertainty in the photometry is purely statistical.

Radio measurements were obtained contemporaneously with the 1980 July observations using the cooled $2.8 \mathrm{~cm}$ amplifier receiver on the $40 \mathrm{~m}$ telescope of the Owens Valley Radio Observatory. A feed system incorporating two identical feeds equally and oppositely offset from the radio axis gave an aperture efficiency of $41 \%$ and a main beam half-power width of 3:3. The observations were calibrated by observing a noise tube which in turn was calibrated daily by observing a set of calibration sources from Baars et al.(1977), including 3C 48, 3C 123, $3 \mathrm{C} 227,3 \mathrm{C} 286$, and $3 \mathrm{C} 348$. The radio data are given in Table 2.

\section{DATA}

\section{a) Infrared Observations}

Only the source $1413+135$, of the four objects examined, was detected at $10 \mu \mathrm{m}$ (Table 1). For the other three objects, $3 \sigma$ upper limits of about $25 \mathrm{mJy}$ were placed on the $10 \mu \mathrm{m}$ flux density.

All four objects were found to vary in brightness at one or more wavelengths. $1413+135$ and $0406+121$ varied at

TABLE 2

$2.8 \mathrm{~cm}$ OBSERVATIONS (1980 Jul 24)

\begin{tabular}{|c|c|c|c|}
\hline Object & $\begin{array}{l}2.8 \mathrm{~cm} \text { Flux } \\
\text { Density (Jy) }\end{array}$ & \multicolumn{2}{|c|}{ Spectral Indices ${ }^{\mathrm{a}} \alpha$} \\
\hline $\begin{array}{l}1413+135 \ldots \ldots \ldots \ldots \ldots \\
2255+41 \ldots \ldots \ldots \ldots \ldots \ldots\end{array}$ & $\begin{array}{l}2.28 \pm 0.11 \\
0.61 \pm 0.09\end{array}$ & $\begin{array}{l}-0.71 \pm 0.03(73-11 \mathrm{~cm}) \\
-0.60 \pm 0.08(11-2.8 \mathrm{~cm})\end{array}$ & $\begin{array}{c}1.60 \pm 0.05(11-2.8 \mathrm{~cm}) \\
\ldots\end{array}$ \\
\hline $0026+34 \ldots \ldots \ldots \ldots \ldots$ & $0.95 \pm 0.03$ & $-0.29 \pm 0.06(11-2.8 \mathrm{~cm})$ & $-0.56 \pm 0.16(2.8-0.95 \mathrm{~cm})$ \\
\hline $0406+121 \ldots \ldots \ldots \ldots \ldots$ & $0.90 \pm 0.04$ & $0.16 \pm 0.08(11-2.8 \mathrm{~cm})$ & \\
\hline
\end{tabular}

${ }^{a}$ Spectral index $\alpha$ defined by $S_{v} \propto v^{\alpha}$.

NoTE.- Additional radio data for $1413+135$ from Hoskins et al. 1972, Condon, Hicks, and Jauncey 1977, and Pauliny-Toth et al. 1978; for 2255 + 41 from Pauliny-Toth et al. 1978; for $0026+34$ from Pauliny-Toth et al. 1978 and Witzel et al. 1978; and for $0406+121$ from Condon, Hicks, and Jauncey 1977. 
$1.25 \mu \mathrm{m}, 1.65 \mu \mathrm{m}$, and $2.2 \mu \mathrm{m}$ by up to a factor of 2 on time scales as rapid as 3 weeks (RLK). Observations on successive nights did not, however, reveal any night-tonight variations in the emission from these two sources. The $2.2 \mu \mathrm{m}$ emission from $2255+41$ and $0026+34$ remained constant over the 2 year period covered by the present observations and those of RLK.

In the cases of $1413+135$ and $0406+12$, an examination of the spectral indices between $1.25 \mu \mathrm{m}$ and $1.65 \mu \mathrm{m}$, (denoted $\alpha_{\mathrm{JH}}$ ) and between $1.65 \mu \mathrm{m}$ and $2.2 \mu \mathrm{m}$ (denoted $\alpha_{\mathrm{HK}}$ ) reveals no statistically significant color variations. For the other two objects, the question of color variations is less easy to answer. In the case of $2255+41$, the data suggest that the $1.65 \mu \mathrm{m}$ flux density doubled and $\alpha_{\mathrm{HK}}$ changed from $\sim-3 \pm 1$ to $\sim-1 \pm 1$ in somewhat less than 3 weeks in 1980 August. As noted above, although care was taken, the $1.25 \mu \mathrm{m}$ and the $1.65 \mu \mathrm{m}$ data are potentially sensitive to contamination by a nearby field star. The apparent change of slope for $0026+34$ is of low statistical significance. Thus the question of spectral variations has not been resolved by these data.

The near-infrared data also show that for $1413+135$, $2255+41$, and $0026+34$ the energy distributions between $1.25 \mu \mathrm{m}$ and $2.2 \mu \mathrm{m}$ can each be described by a single spectral index, with no evidence for curvature. For $0406+121$, the color data, averaged over all of the observations, give $\alpha_{J H}=-2.5 \pm 0.5$ and $\alpha_{H K}=$ $-1.6 \pm 0.2$, suggesting, at the $2 \sigma$ confidence level, that there is a flattening of the spectrum longward of $1.65 \mu \mathrm{m}$. The 1.5-2.4 $\mu \mathrm{m}$ filter wheel spectra (Fig. 1) show that any spectral lines must have equivalent widths less than $30 \mathrm{~nm}$ for $1413+135$ and $90 \mathrm{~nm}$ for $0406+121$.

\section{b) Radio Observations}

The radio spectral indices are included in Table 2 . The spectrum of $1413+135$ is concave, with a minimum lying between $6 \mathrm{~cm}$ and $11 \mathrm{~cm}$. At $85 \mathrm{~cm}$ (Douglas et al. 1980), the source has a flat spectrum and is nonvariable and compact $\left(<10^{\prime \prime}\right)$. At $20 \mathrm{~cm}$ the source is $10 \%$ resolved on the shortest Very Large Array (VLA) baseline, but is unresolved at wavelengths shorter than $6 \mathrm{~cm}$ (VLA calibrator list 1979).

The two sources showing the strongest variations in the infrared, $1413+135$ and $0406+121$, are also variable at short radio wavelengths. In the case of $1413+135,6 \mathrm{~cm}$ data from 1972 (Shimmins, Bolton, and Wall 1975), 1974 (Pauliny-Toth et al. 1978) and 1979 (VLA calibrator list 1979) show $50 \%$ variations over this time period. Douglas et al. (1980) and Hoskins et al. (1972) find no variation in the low-frequency component from 1971-1977. At $11 \mathrm{~cm}$, $0406+121$ varied by up to $30 \%$ over a 3 year period (Condon, Hicks, and Jauncey 1977; Shimmins, Bolton, and Wall 1975). A comparison of our data with those of Pauliny-Toth et al. (1978) shows that neither $2255+41$ nor $0026+34$ varied significantly at $2.8 \mathrm{~cm}$.

\section{DISCUSSION}

\section{a) The Nature of the RLK Sources}

Figure 2 shows that $1413+135$ as well as BL Lac and AO $0235+164$ radiate the bulk of their power at wave-

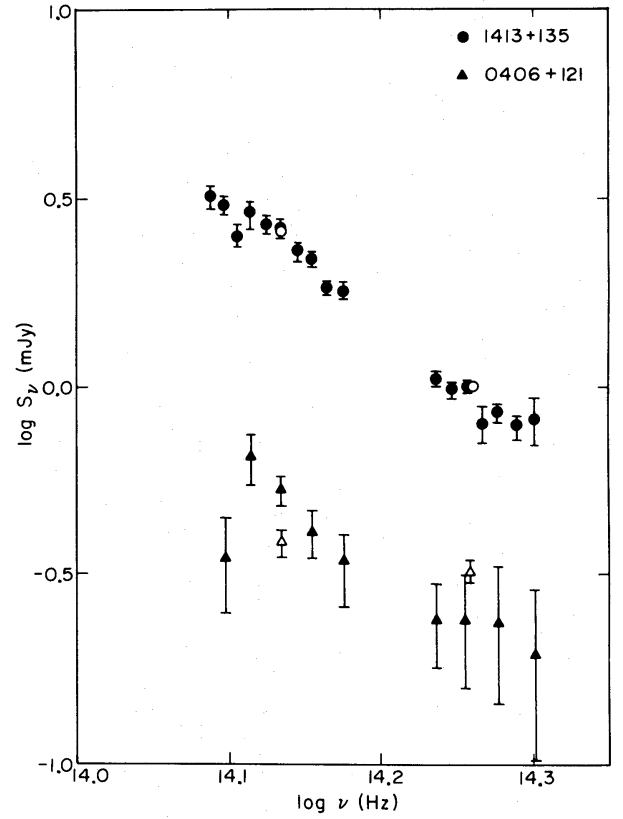

Fig. 1.-A $5 \%$ resolution spectrum of the sources $1413+135$ (circles) and $0406+121$ (triangles) taken on 1980 February 11. Broadband data obtained at the same time are shown with open symbols.

lengths longer than $1 \mu \mathrm{m}$. Typical quasars such as $3 \mathrm{C} 273$ show relatively more emission at visual and ultraviolet wavelengths.

Although BL Lac objects tend to be redder than quasars (Rieke and Kinman 1974), such class distinctions are by no means firm. Smith and Spinrad (1980) have discovered a group of extremely red quasars, while Worrall et al. (1981) have found that X-ray selected BL Lac objects emit most of their power at optical and UV wavelengths rather than in the infrared.

At other wavelengths, $1413+135$ is similar to BL Lac objects or to radio selected quasars. Its $2.8 \mathrm{~cm}$ to $10 \mu \mathrm{m}$ spectral index is comparable to other high-frequency synchrotron sources (Jones and Hardee 1979; Rieke and Lebofsky 1980). The $2 \mathrm{keV}$ flux density from $1413+135$ is $2.2 \times 10^{-8}$ Jy (Bregman et al. 1981; assuming a bandpass of $0.5-3.0 \mathrm{keV}$ and an X-ray spectral index of -1.0 ), giving a spectral index between $10 \mu \mathrm{m}$ and $2 \mathrm{keV}$ of $-1.4 \pm 0.1$. For the six quasars in common between the lists of Tananbaum et al. (1979) and Neugebauer et al. (1979), the same index has a similar value, -1.2 , with a dispersion of 0.2 . The ratio of optical and infrared luminosity to the X-ray luminosity is consistent with either the class of quasars or BL Lac objects (Tananbaum et al. 1979; Schwartz et al. 1979).

In $1413+135$ and $0406+121$ there are brightness variations ( $>99 \%$ confidence) but no slope changes. For $2255+41$ there are potentially variations of slope as well. These findings are similar to those of O'Dell et al. (1978) who found brightness variations in 10 of 13 quasars, BL Lac objects, and Seyfert galaxies examined, but spectral variations in fewer than half that number. 


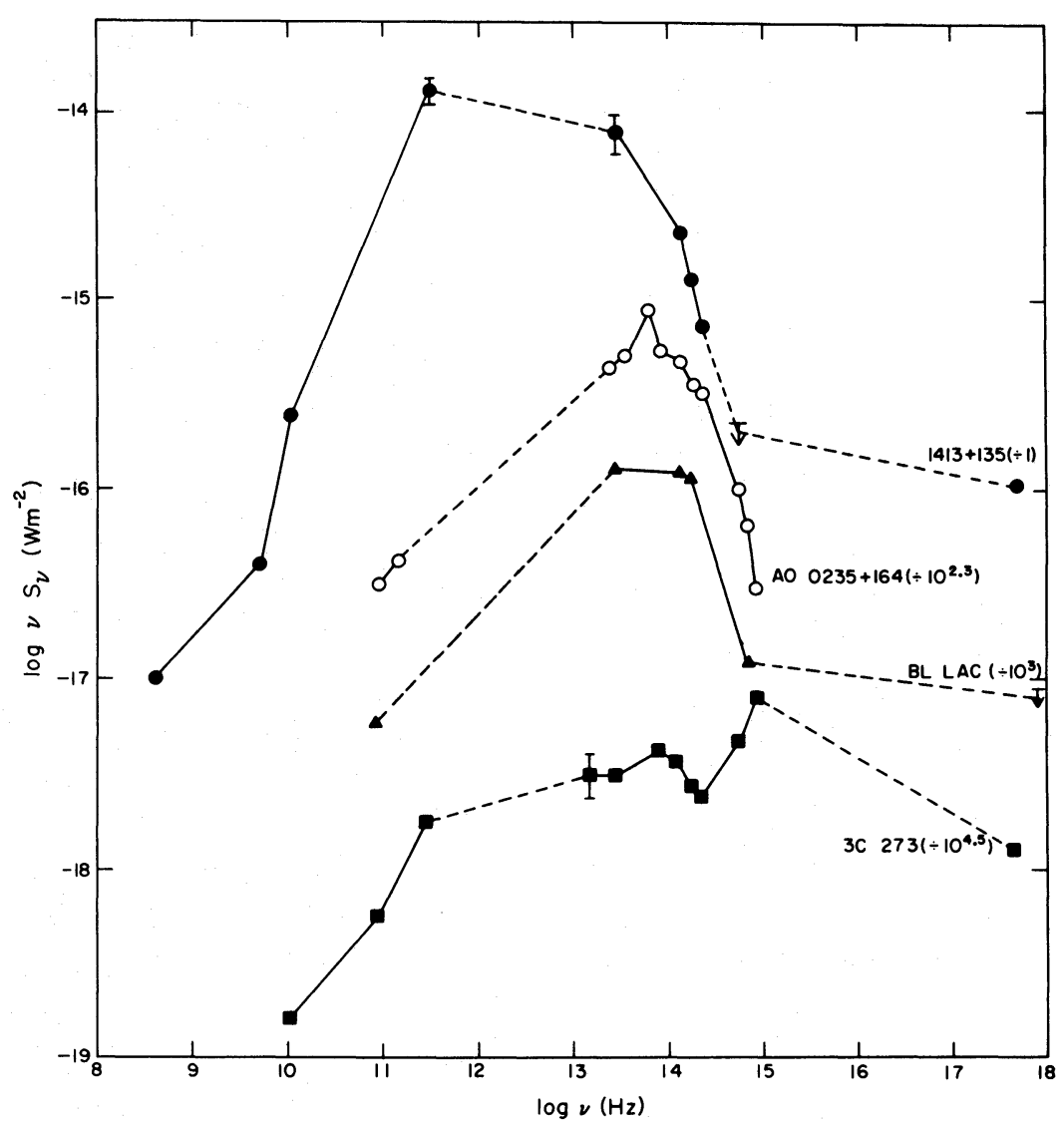

Fig. 2.-Composite energy distributions for $1413+135$, two BL Lac objects (BL Lac and AO $0235+164$ ), and the archetypal quasar 3C 273. For $1413+135$ the radio data are from Hoskins et al. (1972) and Pauliny-Toth et al. (1978), the X-ray datum from Bregman et al. (1981). The millimeter data are from Beichman, Neugebauer, and Soifer (1981). All the observations shown for AO $0235+164$ were obtained on 1975 October 26 and 27 (Rieke et al. 1976). Data for BL Lac are from coordinated observations by Epstein et al. (1972) and from Soifer and Neugebauer (1981). The X-ray upper limit is from Schwartz et al. (1979). Data for 3C 273 come from Jones and Hardee (1979 and references therein), Elias et al. (1978), Neugebauer et al. (1979), and Worrall et al. (1979).

The 1.5-2.4 $\mu \mathrm{m}$ spectrum of $1413+135$ has none of the lines typical of a high-redshift quasar. Table 3 lists a number of spectral lines prominent in quasars, the range of redshift necessary to move a given atomic line into the range of observed wavelengths, the average value of the equivalent width of that line in a number of quasars, and the upper limits to the equivalent width of the line in $1413+135$ and $0406+121$. The observed equivalent widths have been reduced to a rest frame using the redshift necessary to shift the line to $2.0 \mu \mathrm{m}$. For $1413+135$, the data convincingly rule out the presence of $\mathrm{H} \alpha$ or $\mathrm{H} \beta+\left[\mathrm{O}_{\mathrm{III}}\right]$ emission within the indicated redshift ranges. For the other lines, the upper limits are suggestive but not compelling. In the case of $0406+121$, only the presence of $\mathrm{H} \alpha$ emission can be ruled out. The infrared data strongly suggest that $1413+135$ is not a quasar with normal line strengths at redshifts $1.3<z<4$, and that $0406+121$ is not a normal quasar with $1.3<z<2.7$. Aaronson and Boroson (1980) have observed the latter source visually and found no spectral lines between $0.5 \mu \mathrm{m}$ and $0.7 \mu \mathrm{m}$, which rules out redshifts of $0<z<0.4$ and $0.9<z<1.5$.
The brightness variations, the energy distribution, and the lack of spectral features all suggest that $1413+135$ is an extremely red BL Lac object. Similar conclusions can be drawn for $2255+41$ because of its variability and for $0406+121$ because of its variability and lack of infrared or optical (Aaronson and Boroson 1980) spectral features.

\section{b) The Shape of the Infrared Continua}

The low level of the $10 \mu \mathrm{m}$ emission detected from $1413+135$ shows that its energy distribution flattens between 2.2 and $10 \mu \mathrm{m}$. Lebofsky (1981) has reported $3 \mu \mathrm{m}$ and $10 \mu \mathrm{m}$ measurements that show the same effect.

The short wavelength observations taken around the time of the $10 \mu \mathrm{m}$ measurements show no brightness variations, so it seems reasonable to predict a $10 \mu \mathrm{m}$ flux density of $\sim 200 \mathrm{mJy}$ from the $2.2 \mu \mathrm{m}$ flux density and $\alpha_{H K}$. The average $10 \mu \mathrm{m}$ flux density of $29-7 \mathrm{mJy}$ clearly lies well below the predicted value. The observed $2.2-10 \mu \mathrm{m}$ spectral index, $\alpha_{K N}$, has the value $-1.8 \pm 0.2$.

The significance of the $10 \mu \mathrm{m}$ upper limit for $2255+41$ is unclear because of the possible color variations in the 
TABLE 3

UPPER Limits to LiNe EQuivalent WidThS

\begin{tabular}{|c|c|c|c|c|}
\hline \multirow[b]{2}{*}{ LINE } & \multirow[b]{2}{*}{$z$} & \multicolumn{3}{|c|}{ Rest Frame Equivalent Widths ${ }^{\mathrm{a}}$ (nm) } \\
\hline & & quasar avg. & $1413+135$ & $0406+121$ \\
\hline $\mathbf{P} \alpha$ & $-0.1-0.3$ & $12 \pm 5(10)$ & $<28(3 \sigma)$ & $<84(3 \sigma)$ \\
\hline $\mathrm{H} \alpha \ldots \ldots$ & $1.3-2.7$ & $46 \pm 16(38)$ & $<10$ & $<30$ \\
\hline $\mathrm{H} \beta+\left[\mathrm{O}_{\mathrm{III}}\right]$ & $2.1-3.9$ & $14 \pm 5(30)$ & $<7$ & $<22$ \\
\hline Mg II ........ & $5.9-6.4$ & $4 \pm 2(21)$ & $<4$ & $<13$ \\
\hline
\end{tabular}

a Given are the average values for the equivalent width, the dispersion, and (in parentheses) the number of quasars used in obtaining these quantities (Neugebauer et al. 1979; Soifer et al. 1981).

source. If, when observed at $10 \mu \mathrm{m}, 2255+41$ still had the steep, $\alpha_{H K}=-4 \pm 1$, energy distribution as measured only 3 days earlier, the $10 \mu \mathrm{m}$ upper limit is far less than the $175 \mathrm{mJy}$ predicted by extrapolation from the short wavelength data. If, on the other hand, the energy distribution of $2255+41$ had already flattened to $\alpha_{H K}=-1.1 \pm 0.5$, as measured 10 days after the $10 \mu \mathrm{m}$ observation, then the $10 \mu \mathrm{m}$ upper limit lies well above the predicted flux density and does not significantly constrain the spectrum. Neither of the $10 \mu \mathrm{m}$ upper limits to the emission from $0026+34$ or $0406+121$ is stringent enough to ascertain whether or not the energy distributions of these objects flatten longward of $2.2 \mu \mathrm{m}$. The $10 \mu \mathrm{m}$ upper limits permit one to set a limit of $\alpha_{K N}>-2.7$.

As discussed above, the near-infrared observations of $0406+121$ suggest a flattening between 1.25 and $2.2 \mu \mathrm{m}$. This result is consistent with optical measurements of $0406+121$ (Aaronson and Boroson 1980) which show a visual spectral index of 3.8 and suggest a steady flattening of the spectrum to longer wavelengths.

Between $2.2 \mu \mathrm{m}$ and $10 \mu \mathrm{m}, 1413+135$ resembles other quasars and BL Lac objects. In 21 quasars the average observed $\alpha_{K N}=-1.2 \pm 0.2$ (Neugebauer et al. 1979), similar to the value of $-1.8 \pm 0.2$ observed for $1413+135$. At the shorter wavelengths, the steepness of the energy distribution of $1413+135, \alpha_{H K}=-3.5 \pm 0.1$, is far different from those of the same sample of quasars for which the average observed $\alpha_{H K}$ is $-1.5 \pm 0.1$. This steepening of BL Lac energy distributions around the middle-infrared relative to those of quasars has been noted by Rieke and Kinman (1974).

\section{c) The Nature of the Continuum Emission Mechanism}

In the previous section, data were presented which we believe indicate that the sources probably form the extreme red end of a distribution of BL Lac objects in much the same way that the faint 3CR quasars studied by Smith and Spinrad (1980) represent the red end of the distribution of quasars. The importance of these sources is that their extreme nature forces a close examination of the emission mechanisms thought to be operating in the whole class.

The radiation from $1413+135,0406+121$, and $2255+41$ is probably nonthermal in origin. Although the steepness of the near-infrared and optical energy distribution is reminiscent of thermal emission from dust, in the case of $1413+135$ the shape of the $1.2-10 \mu \mathrm{m}$ continuum is too broad to be fitted by any single-temperature blackbody curve. More importantly, the rapid time scale of variability almost certainly excludes a thermal mechanism. The equilibrium blackbody temperature for a grain located a few light weeks away from a $10^{12}-10^{13} L_{\odot}$ source is $4000-6000 \mathrm{~K}$, well above the vaporization temperature for typical grain materials (Salpeter 1974). Lebofsky and Rieke (1980) have made models for Seyfert galaxies invoking variable thermal emission, but the time scale of the variations is 1-2 years, the distance of the grains from the central source about $0.5 \mathrm{pc}$, and the equilibrium temperatures only $1500 \mathrm{~K}$.

If the observed radiation is nonthermal in origin, it is still necessary to account for the steepness of the energy distribution shortward of $2.2 \mu \mathrm{m}$. While a steepening of the spectrum at optical wavelengths is characteristic of some BL Lac objects (Fig. 2), in the RLK objects the steepening occurs at longer wavelengths than in other sources. One possibility is that the emission mechanism in these red objects is not appreciably different from that operating in other BL Lac objects, but rather that the location of the break in the spectrum is moved to longer wavelengths in the RLK sources by their being at a higher redshift than other BL Lac sources. The discovery that quasars of only moderate redshift, $z<1$, can have extremely steep optical continua (Smith and Spinrad 1980) argues against this interpretation and suggests that the radiation from some quasars and BL Lac objects is intrinsically very red.

RLK have discussed the possibility that the lack of high frequency emission is due to a dearth of sufficiently energetic electrons. Future X-ray spectral observations of $1413+135$ can be used to resolve this question. In the synchrotron self-Compton model (e.g., Jones, O'Dell, and Stein 1974) for continuum emission, electrons upconvert a small fraction of their synchrotron photons to X-ray energies. If the optical/infrared radiation represents the high energy end of the synchrotron emission (see also Rudnick et.al. 1978), then breaks in the electron and optical/infrared spectra will cause a break in the X-ray spectrum with a ratio of X-ray to optical/infrared break energies given approximately by $\gamma^{2}$ where $\gamma$ is the electron 
Lorentz factor. For values of $\gamma$ of a few hundred (Jones 1979), this model predicts an X-ray break energy near $10 \mathrm{keV}$.

An alternative possibility is that absorption by dust around the source is responsible for reddening an intrinsically less steep spectrum. To estimate the amount of dust required, assume that the reddening by dust within the Galaxy is negligible for an object at $b^{\mathrm{II}}=65$ (Burstein and Heiles 1978) and that the intrinsic $\alpha_{H K}$ of $1413+135$ is about -1.5 , similar to other quasars (Neugebauer et al. 1979) or BL Lac objects (O'Dell et al. 1978). Then the $\alpha_{H K}=-3.5$ observed for $1413+135$ implies an $H-K$ color excess $E(H-K)=0.6 \mathrm{mag}$. For the standard interstellar reddening curve at zero redshift (Becklin et al. 1978), this color excess corresponds to $A_{v} \sim 8$ mag. Because the effect of redshift is to decrease the $A_{v}$ required to produce a given color excess, the true amount of dust present is likely to be substantially less, e.g., $A_{v} \sim 4 \mathrm{mag}$ for $z=1$. This amount of dust is consistent with the infrared data and with an estimate of the visual magnitude of $1413+135, m_{v} \sim 21-23$ mag, made from the TV guider at the $5 \mathrm{~m}$ telescope.

The question of dust in quasars and BL Lac objects is an open one. Smith and Spinrad (1980) have suggested that very red quasars such as $3 \mathrm{C} 68.1$ could owe their color to about 1-2 mag of internal dust absorption, although these authors point out that the lack of a $2200 \AA$ feature weakens the argument. Soifer et al. (1981) and Puetter et al. (1981) have argued against the presence of substantial amounts of dust on the basis of the hydrogen line ratios observed in a number of quasars. A natural test for the presence of dust is to look at wavelengths longer than $10 \mu \mathrm{m}$ for the reradiation of the energy absorbed by the dust (Ennis et al. 1981). The data of Figure 2 show that any such search must be made at wavelengths between $10 \mu \mathrm{m}$ and $1 \mathrm{~mm}$. Further, if there is a large amount of intervening dust and if, as in the Galaxy, the column density of cool material scales with the dust column density (see, e.g., Ryter, Cesarsky, and Adouze 1975), then the $\mathrm{X}$-ray spectrum will indicate a substantial amount of low energy photoelectric absorption. If, for example, the absorption local to the source corresponds to $A_{V} \sim 4$ mag, the X-ray cutoff energy will be about $1.4 \mathrm{keV}$ at the source.

\section{CONCLUSIONS}

1. The energy distribution of the very red extragalactic radio source $1413+135$ does not continue its steep rise beyond $2.2 \mu \mathrm{m}$. Its spectrum must flatten between 2.2 and $10 \mu \mathrm{m}$. Unless $2255+41$ varied significantly in the 3 days between the near-infrared and $10 \mu \mathrm{m}$ observations, the energy distribution of this object must flatten as well.

2. An overall energy distribution from radio to X-ray wavelengths for $1413+135$ shows that the spectrum of this object is not appreciably different from that of other quasars and BL Lac objects-except for the very steep optical and near-infrared region. The variability and the lack of spectral features suggest that $1413+135$, $2255+41$, and $0406+121$ are BL Lac objects.

3. The steepness of the optical/near-infrared part of the spectrum could be due to an intrinsic lack of high energy electrons or to reddening by dust of a more normal energy distribution. Observations at submillimeter wavelengths or in X-rays should help to clarify this question.

We thank the staffs at Mauna Kea, Palomar, and Owens Valley for their assistance, particularly night assistants Ron Koehler, Barbara Schaeffer, and Juan Carrasco. Dr. J. Bregman communicated X-ray data in advance of publication and offered several useful comments. Drs. G. Rieke and M. Lebofsky kindly provided positions for a number of guide stars near the infrared sources. We thank Dr. Rieke for a critical reading of the manuscript and Dave Ennis for useful discussions. Dr. D. Worrall brought the optical properties of X-ray selected BL Lac objects to our attention. D. Garrett and E. Bozyan communicated $85 \mathrm{~cm}$ data in advance of publication. The work reported here was supported in part by grants from NASA and NSF.

\section{REFERENCES}

Aaronson, M., and Boroson, T. 1980, Nature, 283, 746.

Baars, J. W. M., Genzel, R., Pauliny-Toth, I. I. K., and Witzel, A. 1977, Astr. Ap., 61, 99

Becklin, E. E., Matthews, K., Neugebauer, G., and Willner, S. P. 1978, Ap. J., 220, 831.

Beichman, C. A., Neugebauer, G., Soifer, B. T., Wootten, H. A., Roellig, T., and Harvey, P. M. 1981, Nature, submitted.

Bregman, J. N., Rieke, G. H., Aller, H., Lebofsky, M. J., Aller, M.,

Hodge, P., Glassgold, A. E., Huggins, P. 1981, in preparation.

Burstein, D., and Heiles, C. 1978, Ap. J., 225, 40.

Condon, J. J., Hicks, P. D., and Jauncey, D. L. 1977, A.J., 82, 692

Douglas, J. N., Bash, F. N., Torrence, G. W., and Wolfe, C. 1980, University of Texas Publications in Astronomy, No. 17.

Elias, J. H. et al. 1978, Ap. J., 220, 25.

Elias, J. H., and Neugebauer, G. 1981, in preparation.

Ennis, D. J., Soifer, B. T., Neugebauer, G., and Werner, M. 1981, in preparation.

Epstein, E. E. et al. 1972, Ap. J. (Letters), 178, L51.

Hoskins, D. G., Murdoch, N. S., Hazard, C., and Jauncey, D. L. 1972, Australian J. Phys., 25, 559.
Jones, T. W. 1979, Ap. J., 233, 796.

Jones, T. W., and Hardee, P. E. 1979, Ap. J., 228, 268

Jones, T. W., O'Dell, S. L., and Stein, W. A. 1974, Ap. J., 188, 353.

Lebofsky, M. J. 1981, in IAU Symposium 96, Infrared Astronomy, ed. C. G. Wynn-Williams and D. Cruickshank (Dordrecht: Reidel).

Lebofsky, M. J., and Rieke, G. H. 1980, Nature, 284, 410.

Neugebauer, G., Oke, J. B., Becklin, E. E., and Matthews, K. 1979, Ap. J., 230, 79.

O'Dell, S. L., Puschell, J. J., Stein, W. A., and Warner, J. W. 1978, Ap. J. Suppl., 38, 267.

Pauliny-Toth, I. I. K., Witzel, A., Preuss, E., Kuhr, H., Kellermann, I. K., Fomalont, E. B., and Davis, M. M. 1978, A.J., 83, 451.

Puetter, R. C., Smith, H. E., Willner, S. P., and Pipher, J. L. 1981, preprint.

Rieke, G. H., Grasdalen, G. L., Kinman, T. D., Hintzen, P., Wills, B. J., and Wills, D. 1976, Nature, 260, 754.

Rieke, G. H., and Kinman, T. D. 1974, Ap. J. (Letters), 192, L115.

Rieke, G. H., and Lebofsky, M. J. 1980, in I AU Symposium 92, Objects of High Redshift, ed. G. O. Abell and P. J. E. Peebles (Dordrecht: Reidel), p. 263. 
Rieke, G. H., Lebofsky, M. J., and Kinman, T. D. 1979, Ap. J. (Letters), 232, L151 (RLK)

Iudnick, L., Owen, F. N., Jones, T. W., Puschell, J. J., and Stein, W. A. 1978, Ap. J. (Letters), 225, L5.

Ryter, C., Cesarsky, C. J., and Adouze, J. 1975, Ap. J., 198, 103.

Salpeter, E. E. 1974, Ap. J., 193, 579.

Schwartz, D. A., Doxsey, R. E., Griffiths, R. E., Johnston, M. D., and Schwarz, J. 1979, Ap. J. (Letters), 229, L53.

Shimmins, A. J., Bolton, J. G., and Wall, J. V. 1975, Australian J. Phys. Suppl., 34, 63.

Smith, H. E., and Spinrad, H. 1980, Ap. J., 236, 419.
Soifer, B. T., and Neugebauer, G. 1981, in I AU Symposium 96, Infrared Astronomy, ed. C. G. Wynn-Williams and D. Cruickshank (Dordrecht: Reidel).

Soifer, B. T., Neugebauer, G., Oke, J. B., and Matthews, K. 1981, Ap. J., 243, 369.

Tananbaum, H. et al. 1979, Ap. J. (Letters), 234, L9.

Witzel, A., Pauliny-Toth, I. I. K., Geldzahler, B. J., and Kellermann, I. K. 1978, A.J., 83, 475.

Worrall, D. M., Boldt, E. A., Holt, S. S., Mushotzky, R. F., and Serlemitsos, P. J. 1981, Ap. J., 243, 53.

Worrall, D. M., Mushotzky, R. F., Boldt, E. A., Holt, S. S., and Serlemitsos, P. J. 1979, Ap. J., 232, 683.

C. A. Beichman, S. H. Pravdo, G. Neugebauer, B. T. Soifer, and K. Matthews: 320-47, California Institute of Technology, Pasadena, CA 91125

H. A. Wootten: 102-24, California Institute of Technology, Pasadena, CA 91125 